\title{
AN ISOPERIMETRIC RESULT FOR THE FUNDAMENTAL FREQUENCY VIA DOMAIN DERIVATIVE.
}

\author{
CARLO Nitsch \\ Dipartimento di Matematica e Applicazioni "R. Caccioppoli" \\ Complesso Monte S. Angelo \\ Via Cintia, 80126 Napoli, Italy
}

\begin{abstract}
The Faber-Krahn deficit $\delta \lambda$ of an open bounded set $\Omega$ is the normalized gap between the values that the first Dirichlet Laplacian eigenvalue achieves on $\Omega$ and on the ball having same measure as $\Omega$. For any given family of open bounded sets of $\mathbb{R}^{N}(N \geq 2)$ smoothly converging to a ball, it is well known that both $\delta \lambda$ and the isoperimetric deficit $\delta P$ are vanishing quantities. It is known as well that, at least for convex sets, the ratio $\frac{\delta P}{\delta \lambda}$ is bounded by below by some positive constant (see [3, 19]), and in this note, using the technique of the shape derivative, we provide the explicit optimal lower bound of such a ratio as $\delta P$ goes to zero.
\end{abstract}

\section{INTRODUCTION}

Given an open bounded set of $\mathbb{R}^{N}$ its first Dirichlet Laplacian eigenvalue $\lambda$ is the least positive number for which the boundary problem

$$
\begin{cases}-\Delta u=\lambda u & \text { in } \Omega \\ u=0 & \text { on } \partial \Omega\end{cases}
$$

admits nontrivial solutions.

The Faber-Krahn inequality is a remarkable property which, answering to a conjecture formulated by Lord Rayleight, states that among sets of given measure the ball has the least first Dirichlet Laplacian eigenvalue. Namely if $\Omega^{\sharp}$ denotes the ball having the same measure as $\Omega$ then

$$
\lambda(\Omega) \geq \lambda\left(\Omega^{\sharp}\right) .
$$

Inequality (1) falls in the large class of so-called isoperimetric inequalities. By antonomasia the isoperimetric inequality is the one which characterizes the ball as the set having minimial perimeter among those sets of fixed volume, but nowadays, in a broad sense isoperimetric inequality is an inequality where a functional is optimized under some geometrical prescription. The study of isoperimetric inequalities goes back to the beginning of mathematics and has always been a flourishing field. Recently many authors turned the attention to the study of quantitative versions of the classical isoperimetric inequalities (see for instance $[2,5,6,7,8,9,10]$ ), and quantitative versions of Faber-Krahn inequality have been investigated for instance

1991 Mathematics Subject Classification. Primary: 35P15; Secondary: 49R05, 35J25.

Key words and phrases. Domain derivative; first laplacian eigenvalue; isoperimetric deficit; 
in $[11,16]$. Here we are interested in a recent result [3] obtained in the wake of a celebrated paper by L. E. Payne and H. F. Weinberger [19].

As custom let us denote by

$$
\delta P(\Omega)=\frac{\operatorname{Per}(\Omega)}{\operatorname{Per}\left(\Omega^{\sharp}\right)}-1,
$$

the isoperimetric deficit of $\Omega$ and following [11] we denote by

$$
\delta \lambda(\Omega)=\frac{\lambda(\Omega)}{\lambda\left(\Omega^{\sharp}\right)}-1,
$$

the Faber-Krahn deficit.

The classical isoperimetric inequality and the Faber-Krahn inequality respectively infer that both $\delta P$ and $\delta \lambda$ are always non negative quantities.

When $\Omega$ is convex then (1) can be improved (see [3, 19]) establishing that for any $\eta>0$ there exists $C>0$ depending on $N$ such that if $\delta P(\Omega) \leq \eta$ then

$$
\delta P(\Omega) \geq C \delta \lambda(\Omega) .
$$

The name "quantitative Faber-Krahn inequality" comes from the fact that it quantifies how "small" is the Faber-Krahn deficit when the set $\Omega$ is "close" to the ball having same measure.

It is easy to show that inequality (2) is optimal in the sense that for any $\eta, C, \gamma>0$ there exists a bounded convex set $\Omega$ such that $\delta P(\Omega) \leq \eta$ and $\delta P(\Omega)<C \delta \lambda(\Omega)^{1+\gamma}$.

Very little is known about the optimal value of the constant $C$ even in the limit as $\delta P \rightarrow 0$ and here comes the idea to exploit the technique of shape derivative to investigate the behavior of the ratio $\delta P / \delta \lambda$ along an arbitrary family of sets which converges in a suitable way to a ball. More precisely we use the following definition

Definition 1.1. We say that a one parameter family $\Omega(t)$ of open bounded sets of $\mathbb{R}^{N}$ smoothly converges to an open bounded set $\Omega$ as $t$ goes to zero, if there exists a positive $\delta$, and a one parameter family of transformations $\Phi_{t}(0 \leq t<\delta)$ of $\mathbb{R}^{N}$ in itself such that

(a) $\Phi_{t}$ and $\Phi_{t}^{-1}$ belong to $C^{\infty}\left(\mathbb{R}^{N} ; \mathbb{R}^{N}\right)$ for all $0 \leq t<\delta$;

(b) the mappings $t \rightarrow \Phi_{t}(x)$ and $t \rightarrow \Phi_{t}^{-1}(x)$ belong to $C^{\infty}\left(\left[0, \delta[)\right.\right.$ for all $x \in \mathbb{R}^{N}$;

(c) $\Omega(0)=\Omega$ and $\Omega(t)=\Phi_{t}(\Omega)$ for all $0 \leq t<\delta$;

We denote by $\omega_{N}$ the volume of the unit ball of $\mathbb{R}^{N}$, by $J_{\nu}$ the Bessel function of first kind and order $\nu$, and by $j_{\nu}$ the first positive zero of $J_{\nu}$. Our main result follows.

Theorem 1.2. For any $N \geq 2$ there exists a dimensional constant

$$
C_{N}=\frac{N(N+1) \int_{0}^{j_{N / 2-1}} r J_{N / 2-1}^{2}(r) d r}{2\left(j_{N / 2-1} J_{N / 2-1}^{\prime}\left(j_{N / 2-1}\right)\right)^{2}\left(j_{N / 2-1}^{2}-N\right)}
$$

such that for any given one parameter family of sets $\Omega(t)$ of $\mathbb{R}^{N}$, smoothly converging to a ball as $t \rightarrow 0$, then

$$
\liminf _{t \rightarrow 0} \frac{\delta P(\Omega(t))}{\delta \lambda(\Omega(t))} \geq C_{N} .
$$

The constant $C_{N}$ is optimal and there exists a family $\Omega(t)$ for which the equality sign is achieved. 


\section{Proof of Theorem 1.2}

We consider a family of open bounded sets $\Omega(t)(0 \leq t<\delta)$ smoothly converging as $t \rightarrow 0$ in the sense of Definition 1.1 to a smooth open bounded connected set $\Omega$. For any $0 \leq t<\delta$ we denote by $\lambda(t)$ and $u(x, t)$ respectively the first Dirichlet Laplacian eigenvalue and the normalized solution to

$$
\begin{cases}-\Delta u(x, t)=\lambda(t) u(x, t) & \text { in } \quad \Omega(t) \\ u \geq 0 & \text { in } \quad \Omega(t) \\ u(x, t)=0 & \text { on } \partial \Omega(t) \\ \|u\|_{L^{2}(\Omega(t))}=1 . & \end{cases}
$$

The proof of Theorem 1.2 will be carried on by choosing an arbitrary family of smooth sets converging to a ball as $t$ goes to zero and by performing Taylor expansion of the ratio $\frac{\delta P(\Omega(t))}{\delta \lambda(\Omega(t))}$ around $t=0$. For the seek of simplicity we split the proof of Theorem 1.2 in several steps. In the first step we provide the general expression of first and second order derivatives of the first Dirichlet Laplacian eigenvalue along the family $\Omega(t)$. In the second step we let $\Omega$ be the a ball of $\mathbb{R}^{N}$ and we differentiate $\delta P(\Omega(t))$ and $\delta \lambda(\Omega(t))$ twice at $t=0$ deducing that there exists a functional $\mathcal{F}$ on $C^{\infty}(\partial \Omega)$ such that $\frac{\delta P(\Omega(t))}{\delta \lambda(\Omega(t))}=\mathcal{F}\left(\left.n \cdot \frac{\partial \Phi_{t}}{\partial t}\right|_{t=0}\right)+o(1)$ as $t$ goes to zero. Here $n$ denotes the unit outer normal to $\partial \Omega$. In the third and last step we show that $C_{N}$ is exactly the minimum achieved by the functional $\mathcal{F}$ when we vary $\Phi_{t}$ on the whole class of admissible smooth transformations (in the sense of Definition 1.1 (a)-(b)-(c)).

Step 1. We begin the proof computing the first and the second order derivatives of the Dirichlet Laplacian eigenvalue along the family $\Omega(t)$, using the well known Hadamard's formula. Namely we prove the following Lemma

Lemma 2.1. For $0 \leq t<\delta$ let $u(x, t)$ be the family of solutions to (3) and let $\lambda(t)$ be the corresponding family of eigenvalues. There exists $\varepsilon>0$ such that for all $0 \leq t<\varepsilon$ the family $\lambda(t)$ is smooth and it holds

$$
\begin{aligned}
\lambda^{\prime}(t)= & \int_{\partial \Omega(t)}|D u| \frac{\partial \Phi_{t}}{\partial t}\left(\Phi_{t}^{-1}\right) \cdot D u d \mathcal{H}^{N-1}, \\
\lambda^{\prime \prime}(t)= & \int_{\partial \Omega(t)}|D u|\left(\left[\frac{\partial \Phi_{t}}{\partial t}\left(\Phi_{t}^{-1}\right)\right]^{T} \cdot D^{2} u \cdot \frac{\partial \Phi_{t}}{\partial t}\left(\Phi_{t}^{-1}\right)\right. \\
& \left.+D u \cdot \frac{\partial^{2} \Phi_{t}}{\partial t^{2}}\left(\Phi_{t}^{-1}\right)+2 D w \cdot \frac{\partial \Phi_{t}}{\partial t}\left(\Phi_{t}^{-1}\right)\right) d \mathcal{H}^{N-1}
\end{aligned}
$$


where $w$ solves

$$
\begin{cases}-\Delta w(x, t)=\lambda^{\prime}(t) u(x, t)+\lambda(t) w(x, t) & x \in \Omega(t) \\ w(x, t)=-\frac{\partial \Phi_{t}}{\partial t}\left(\Phi_{t}^{-1}(x)\right) \cdot D u(x, t) & x \in \partial \Omega(t) . \\ \int_{\Omega(t)} u w=0 & \end{cases}
$$

Equations (4)-(5)-(6) are related to other formulas which can be found in literature (see $[13,14,18,21]$ and the references therein contained). In particular $[14,18]$ contain very general formulation of the notion of shape derivative with application to the Dirichlet Laplacian eigenvalues. We also observe that very often in literature (see for instance [13, Theorem 2.5.6]) it is considered the case of a first order perturbations of identity, namely $\Phi_{t}=\mathbb{I}+t W$, where $W$ is a suitably smooth vector field. The result is that the term $D u \cdot \frac{\partial^{2} \Phi_{t}}{\partial t^{2}}$ in (5), which in our case plays a crucial rule, would be missing.

Hence, for the seek of completeness we decided to provide here a complete proof of the statement of Lemma 2.1, and we exploit a level sets method.

Proof of Lemma 2.1. Classical regularity theory [12] for elliptic equation ensures that, for all $t \in\left[0, \delta\left[, u(x, t) \in C^{\infty}(\bar{\Omega}(t))\right.\right.$. Moreover, arguing as in [14, Chapter 5], there exists at least some positive $\varepsilon<\delta$ such that the function $u(x, t)$ belongs to $C^{\infty}\left(\left[0, \varepsilon\left[; C^{\infty}(\bar{\Omega}(t))\right)\right.\right.$.

Differentiating with respect to $t$ the equation in (3) we get

$$
-\Delta \frac{\partial u}{\partial t}=\lambda^{\prime}(t) u+\lambda(t) \frac{\partial u}{\partial t}
$$

Since $\Omega(t)$ is, at any time, the zero-level set of $u(x, t)$, if $y \in \partial \Omega$ then $\Phi_{t}(y) \in \partial \Omega(t)$ and we have

$$
u\left(\Phi_{t}(y), t\right)=0 \quad \text { for all } y \in \partial \Omega \text { and } 0<t<\varepsilon .
$$

Now, the boundary point $\Phi_{t}(y) \in \partial \Omega(t)$ moves with velocity $\frac{\partial \Phi_{t}(y)}{\partial t}$. Differentiating once (8) with respect to $t$ we get

$$
\frac{d}{d t} u\left(\Phi_{t}(y), t\right)=D u\left(\Phi_{t}(y), t\right) \cdot \frac{\partial \Phi_{t}}{\partial t}(y)+\frac{\partial u}{\partial t}\left(\Phi_{t}(y), t\right)=0,
$$

and hence

$$
D u \cdot \frac{\partial \Phi_{t}}{\partial t}\left(\Phi_{t}^{-1}\right)+\frac{\partial u}{\partial t}=0 \quad \text { on } \partial \Omega(t) .
$$

Therefore the projection of the velocity $\frac{\partial \Phi_{t}}{\partial t}(y)$ along the direction of the unit outer normal $n$ is equal to $\frac{1}{|D u|} \frac{\partial u}{\partial t}$, which is pointwise defined since $\Omega(t)$ is smooth and standard barrier arguments imply that $D u$ does not vanish on $\partial \Omega(t)$.

Differentiating twice (8) with respect to $t$ we get

$$
\left[\frac{\partial \Phi_{t}}{\partial t}\left(\Phi_{t}^{-1}\right)\right]^{T} \cdot D^{2} u \cdot \frac{\partial \Phi_{t}}{\partial t}\left(\Phi_{t}^{-1}\right)+D u \cdot \frac{\partial^{2} \Phi_{t}}{\partial t^{2}}\left(\Phi_{t}^{-1}\right)+2\left(D \frac{\partial u}{\partial t}\right) \cdot \frac{\partial \Phi_{t}}{\partial t}\left(\Phi_{t}{ }^{-1}\right)+\frac{\partial^{2} u}{\partial t^{2}}=0
$$

which highlights the connection between the acceleration $\frac{\partial^{2} \Phi_{t}}{\partial t^{2}}$ of a boundary point $x \equiv \Phi_{t}(y) \in \partial \Omega(t)$ and the value of $\frac{\partial^{2} u}{\partial t^{2}}$ at the same point. 
Now, if $f(x, t)$ is a smooth function and $J(t)=\int_{\Omega(t)} f(x, t) d x$, the classical Hadamard formula gives (see, for instance, $[14,21]$ )

$$
J^{\prime}(t)=\int_{\Omega(t)} \frac{\partial f}{\partial}(x, t) d x+\int_{\partial \Omega(t)} f(x, t) \frac{1}{|D u|} \frac{\partial u}{\partial t} d \mathcal{H}^{N-1} .
$$

Therefore, since the $L^{2}$ norm of $u$ is constant with respect to $t$ and $u$ vanishes on $\partial \Omega(t)$ we have

$$
\begin{aligned}
& \frac{d}{d t} \int_{\Omega(t)} u^{2} d x=2 \int_{\Omega(t)} u \frac{\partial u}{\partial t} d x=0 \\
& \frac{d^{2}}{d t^{2}} \int_{\Omega(t)} u^{2} d x=2 \int_{\Omega(t)} u \frac{\partial^{2} u}{\partial t^{2}} d x+2 \int_{\Omega(t)}\left(\frac{\partial u}{\partial t}\right)^{2} d x=0 .
\end{aligned}
$$

Furthermore (11) applied to $\lambda(t)$ provides the relation

$\lambda^{\prime}(t)=\frac{d}{d t} \int_{\Omega(t)}|D u|^{2} d x=-\int_{\partial \Omega(t)}|D u| \frac{\partial u}{\partial t} d \mathcal{H}^{N-1}=\int_{\partial \Omega(t)}|D u| \frac{\partial \Phi_{t}}{\partial t}\left(\Phi_{t}{ }^{-1}\right) \cdot D u d \mathcal{H}^{N-1}$,

or equivalently

$$
\lambda^{\prime}(t)=\int_{\Omega(t)} D u D\left(\frac{\partial u}{\partial t}\right) d x
$$

obtaining (4).

Finally, if we differentiate $\lambda$ twice, we can use (7),(12),(13) and (14) to get

$$
\begin{aligned}
\lambda^{\prime \prime}(t) & =\frac{d}{d t} \int_{\Omega(t)} D u D\left(\frac{\partial u}{\partial t}\right) d x \\
& =\int_{\Omega(t)}\left|D \frac{\partial u}{\partial t}\right|^{2} d x+\int_{\Omega(t)} D u D \frac{\partial^{2} u}{\partial t^{2}} d x+\int_{\partial \Omega(t)} \frac{D u}{|D u|}\left(D \frac{\partial u}{\partial t}\right) \frac{\partial u}{\partial t} d \mathcal{H}^{N-1} \\
& =-\int_{\Omega(t)}\left(\Delta \frac{\partial u}{\partial t}\right) \frac{\partial u}{\partial t} d x-\int_{\Omega(t)} \Delta u \frac{\partial^{2} u}{\partial t^{2}} d x-\int_{\partial \Omega(t)}|D u| \frac{\partial^{2} u}{\partial t^{2}} d \mathcal{H}^{N-1} \\
& =\lambda(t) \int_{\Omega(t)}\left(\left(\frac{\partial u}{\partial t}\right)^{2}+u \frac{\partial^{2} u}{\partial t^{2}}\right) d x+\lambda^{\prime}(t) \int_{\Omega(t)} u \frac{\partial u}{\partial t} d x-\int_{\partial \Omega(t)}|D u| \frac{\partial^{2} u}{\partial t^{2}} d \mathcal{H}^{N-1} \\
& =-\int_{\partial \Omega(t)}|D u| \frac{\partial^{2} u}{\partial t^{2}} d \mathcal{H}^{N-1} .
\end{aligned}
$$

Once we set

$$
w(x, t)=\frac{\partial u}{\partial t}(x, t)
$$

in (7), (9) and (12), using (10) we get (5) and the proof is complete

We observe that the family of transformations $\Phi_{t}$ is not uniquely determined by the family $\Omega(t)$. In particular it is always possible to choose the velocity vector field 
$\left.\frac{\partial \Phi_{t}}{\partial t}\right|_{t=0}$ orthogonal to $\partial \Omega$. In such a case (4)-(5)-(6) computed at $t=0$ become

$$
\begin{aligned}
& \lambda^{\prime}(0)=-\left.\int_{\partial \Omega}|D u|^{2} n \cdot \frac{\partial \Phi_{t}}{\partial t}\right|_{t=0} \\
& \lambda^{\prime \prime}(0)=\int_{\partial \Omega}\left[\omega^{2} H-\left.|D u|^{2} n \cdot \frac{\partial^{2} \Phi_{t}}{\partial t^{2}}\right|_{t=0}-2 \omega \frac{\partial \omega}{\partial n}\right] d \mathcal{H}^{N-1}, \\
& \\
& \left\{\begin{array}{l}
-\Delta \omega(x)=\lambda^{\prime}(0) u(x, 0)+\lambda(0) \omega(x) \quad \text { in } \Omega \\
\int_{\Omega} u(x, 0) \omega(x) d x=0
\end{array}\right.
\end{aligned}
$$

Here $H$ is the sum of the principal curvatures of $\partial \Omega$ and $n$ the unit outer normal of $\partial \Omega$. Since $\Delta u(x, 0)$ vanishes on $\partial \Omega$ we have used the identity

$$
D u^{T} \cdot D^{2} u \cdot D u=-\operatorname{div}\left(\frac{D u}{|D u|}\right)|D u|^{3} \quad \text { on } \partial \Omega,
$$

in conjuction with $-\operatorname{div}\left(\frac{D u}{|D u|}\right)=H$.

Step 2. Due to the invariance of both isoperimetric and Faber-Krahn deficits with respect to homotheties we shall perform all the remaining computation under the assumption that the family $\Omega(t)$ has constant volume in $t$ equal to $\omega_{N}$, therefore from now on $\Omega \equiv \Omega(0)$ is just a unit ball in $\mathbb{R}^{n}$.

Without loss of generality, we also assume that the velocity field $\left.\frac{\partial \Phi_{t}}{\partial t}\right|_{t=0}$ is orthogonal to $\partial \Omega$ and for all $x \in \partial \Omega$ we denote by $V(x)=\left.n(x) \cdot \frac{\partial \Phi_{t}}{\partial t}\right|_{t=0}$ and by $A(x)=\left.n(x) \cdot \frac{\partial^{2} \Phi_{t}}{\partial t^{2}}\right|_{t=0}$ respectively the initial scalar velocity and the projection of the initial acceleration along the unit outer normal $n(x)$ of $\Omega$.

Under these assumptions, for $t$ small enough, the boundary of $\Omega(t)$ can be represented in polar coordinates $r \in \mathbb{R}^{+}, \xi \in \mathcal{S}^{N-1}$ by an equation

$$
r(\xi, t)=1+V(\xi) t+\frac{A(\xi)}{2} t^{2}+o\left(t^{2}\right) .
$$

If $\sigma_{\xi}$ denotes the usual surface area measure on $\mathcal{S}^{N-1}$ then

$$
\operatorname{Per}(\Omega(t))=\int_{\mathcal{S}^{N-1}} r(\xi, t)^{N-2} \sqrt{r(\xi, t)^{2}+\left|D_{\xi} r(\xi, t)\right|^{2}} d \sigma_{\xi},
$$

and after a taylor expansion we have

$$
\begin{aligned}
\operatorname{Per}(\Omega(t))= & n \omega_{N}+t \int_{\mathcal{S}^{N-1}}(N-1) V(\xi) d \sigma_{\xi} \\
& +\frac{t^{2}}{2} \int_{\mathcal{S}^{N-1}}\left[(N-1) A(\xi)+(N-1)(N-2) V^{2}(\xi)+\left|D_{\xi} V(\xi)\right|^{2}\right] d \sigma_{\xi}+o\left(t^{2}\right) .
\end{aligned}
$$

On the other hand, since

$$
\omega_{N}=|\Omega(t)| \quad \text { for all } t \in[0, \delta[
$$


then

$$
\begin{aligned}
|\Omega(t)| & =\frac{1}{N} \int_{\mathcal{S}^{N-1}} r(\xi, t)^{N} d \sigma_{\xi} \\
& =\omega_{N}+t \int_{\mathcal{S}^{N-1}} V(\xi) d \sigma_{\xi}+\frac{t^{2}}{2} \int_{\mathcal{S}^{N-1}}\left[A(\xi)+(N-1) V^{2}(\xi)\right] d \sigma_{\xi}+o\left(t^{2}\right)
\end{aligned}
$$

yields

$$
\int_{\mathcal{S}^{N-1}} V(\xi) d \sigma_{\xi}=\int_{\mathcal{S}^{N-1}}\left[A(\xi)+(N-1) V^{2}(\xi)\right] d \sigma_{\xi}=0 .
$$

As a consequence

$$
\operatorname{Per}(\Omega(t))=n \omega_{N}+\frac{t^{2}}{2} \int_{\mathcal{S}^{N-1}}\left[\left|D_{\xi} V(\xi)\right|^{2}-(N-1) V^{2}(\xi)\right] d \sigma_{\xi}+o\left(t^{2}\right)
$$

and

$$
\begin{aligned}
\delta P(\Omega(t)) & =\frac{\operatorname{Per}(\Omega(t))}{N \omega_{N}^{1 / n}|\Omega(t)|^{(N-1) / N}}-1 \\
& =\frac{t^{2}}{2 N \omega_{N}} \int_{\mathcal{S}^{N-1}}\left[\left|D_{\xi} V(\xi)\right|^{2}-(N-1) V^{2}(\xi)\right] d \sigma_{\xi}+o\left(t^{2}\right) .
\end{aligned}
$$

We consider now the series expansion

$$
\lambda(t)=\lambda(0)+t \lambda^{\prime}(0)+\frac{t^{2}}{2} \lambda^{\prime \prime}(0)+o\left(t^{2}\right) .
$$

The gradient $D u(\cdot, 0)$ on $\partial \Omega$ has constant modulus (see [15])

$$
G_{N}=\frac{j_{N / 2-1}^{2} J_{N / 2-1}^{\prime}\left(j_{N / 2-1}\right)}{\left(N \omega_{N} \int_{0}^{j_{N / 2-1}} r J_{N / 2-1}^{2}(r) d r\right)^{1 / 2}},
$$

and therefore using (16) and (19) we deduce $\lambda^{\prime}(0)=0$ in accordance with the fact that the ball, among sets of fixed measure, is a stationary point for the first Dirichlet Laplacian eigenvalue.

Thereafter, for all $x \equiv(r, \xi) \in \Omega$, we set

$$
v(r, \xi)=G_{N} \omega(x)
$$

where $\omega$ is defined in (18).

Taking into account that, for the unit ball, the sum of the principal curvatures $H$ equals $N-1$, from (17) and (19) we get

$$
\lambda^{\prime \prime}(0)=2 G_{N}^{2} \int_{\mathcal{S}^{N-1}}\left[\left.V(\xi) \frac{\partial v(r, \xi)}{\partial r}\right|_{r=1}+(N-1) V^{2}(\xi)\right] d \sigma_{\xi} .
$$

Consequently

$$
\begin{aligned}
\delta \lambda(\Omega(t)) & =\frac{\lambda(t)}{\lambda(0)}-1 \\
& =t^{2}\left(\frac{G_{N}}{j_{N / 2-1}}\right)^{2} \int_{\mathcal{S}^{N-1}}\left[\left.V(\xi) \frac{\partial v(r, \xi)}{\partial r}\right|_{r=1}+(N-1) V^{2}(\xi)\right] d \sigma_{\xi}+o\left(t^{2}\right) .
\end{aligned}
$$

Here we have used the fact that the first Dirichlet Laplacian eigenvalue on the unit ball of $\mathbb{R}^{N}$ is $\lambda(0)=j_{N / 2-1}^{2}$ (see for instance [15]) and that $\lambda^{\prime}(0)=0$. 
We need now an explicit representation of the function $v(r, \xi)$ in terms of the scalar velocity $V(\xi)$. To this aim, we observe that (18), in conjunction with $\lambda^{\prime}(0)=0$ and $\left.|D u(x, 0)|\right|_{\partial \Omega}=G_{N}$ imply that $v(r, \xi)$ satisfies

$$
\begin{cases}-r^{1-N} \frac{\partial}{\partial r}\left(r^{N-1} \frac{\partial v}{\partial r}\right)-r^{-2} \Delta_{\xi} v=j_{N / 2-1}^{2} v & (r, \xi) \in(0,1) \times \mathcal{S}^{N-1} \\ v(1, \xi)=G_{N} V(\xi) & \xi \in \mathcal{S}^{N-1}\end{cases}
$$

where $\Delta_{\xi}$ is the Laplace Beltrami operator on $\mathcal{S}^{N-1}$.

Then we remind (see for instance [17]) that $V(\xi)$ admits an expansion

$$
V(\xi)=\sum_{k=0}^{+\infty} a_{k} Y_{k}(\xi) \quad \xi \in \mathcal{S}^{N-1}
$$

in terms of a family of spherical harmonics $\left\{Y_{k}(\xi)\right\}_{k \in \mathbb{N}}$ which satisfy for all $k \geq 0$

$$
-\Delta_{\xi} Y_{k}=k(k+N-2) Y_{k} \text { and }\left\|Y_{k}\right\|_{L^{2}}=1 .
$$

The coefficient $a_{k}$ is the projection of $V$ on the normalized eigenfuntion $Y_{k}$

$$
a_{k}=\int_{\mathcal{S}^{N-1}} V(\xi) Y_{k}(\xi) d \sigma_{\xi},
$$

so that

$$
\|V\|_{L^{2}}^{2}=\sum_{k=0}^{+\infty} a_{k}^{2}
$$

Notice that $Y_{0}=\left(N \omega_{N}\right)^{-1 / 2}$ and (19) imply

$$
a_{0}=\int_{\mathcal{S}^{N-1}} V Y_{0} d \sigma_{\xi}=\left(N \omega_{N}\right)^{-1 / 2} \int_{\mathcal{S}^{N-1}} V d \sigma_{\xi}=0 .
$$

Accordingly we use the separation of variables $v(r, \xi)=\sum_{k} R_{k}(r) Y_{k}(\xi)$ to solve the Poisson problem (20) and infer

$$
v(r, \xi)=r^{1-N / 2} \sum_{k \geq 1} a_{k} \frac{J_{\ell_{k}}\left(j_{N / 2-1} r\right)}{J_{\ell_{k}}\left(j_{N / 2-1}\right)} Y_{k}(\xi)
$$

where $\ell_{k}=\sqrt{k(k+N-2)+(N / 2-1)^{2}}=k+N / 2-1$.

Consequently we have

$$
\frac{\partial v}{\partial r}(1, \xi)=\sum_{k \geq 1}\left[\left(1-\frac{N}{2}\right)+j_{N / 2-1} \frac{J_{\ell_{k}}^{\prime}\left(j_{N / 2-1}\right)}{J_{\ell_{k}}\left(j_{N / 2-1}\right)}\right] a_{k} Y_{k}(\xi),
$$

and in view of the recurrence relations of the Bessel functions (see $[1, \S 9.1 .27]$ )

$$
J_{\ell}^{\prime}(s)=\frac{\ell}{s} J_{\ell}(s)-J_{\ell+1}(s)
$$

we can write

$$
\frac{\partial v}{\partial r}(1, \xi)=\sum_{k \geq 1}\left[k-j_{N / 2-1} \frac{J_{\ell_{k}+1}\left(j_{N / 2-1}\right)}{J_{\ell_{k}}\left(j_{N / 2-1}\right)}\right] a_{k} Y_{k}(\xi) .
$$

Finally we obtain 


$$
\frac{\delta P(\Omega(t))}{\delta \lambda(\Omega(t))}=\left(\frac{j_{N / 2-1}^{2}}{2 N \omega_{N} G_{N}^{2}}\right) \frac{\sum_{k \geq 2} a_{k}^{2}\left[\ell_{k}^{2}-\frac{N^{2}}{4}\right]}{\sum_{k \geq 2} a_{k}^{2}\left[\ell_{k}+\frac{N}{2}-j_{N / 2-1} \frac{J_{\ell_{k}+1}\left(j_{N / 2-1}\right)}{J_{\ell_{k}}\left(j_{N / 2-1}\right)}\right]}+o(1)
$$

Observe that $a_{1}$ provides no contribution in the summation, indeed the projection of $V$ on the subspace $Y_{1}$ corresponds to a translation of the ball $\Omega(0)$ (with no deformation).

Step 3. It is evident that

$$
\liminf _{t \rightarrow 0} \frac{\delta P(\Omega(t))}{\delta \lambda(\Omega(t))} \geq \inf _{k \geq 2}\left[\left(\frac{j_{N / 2-1}^{2}}{2 N \omega_{N} G_{N}^{2}}\right) \frac{\ell_{k}^{2}-\frac{N^{2}}{4}}{\ell_{k}+\frac{N}{2}-j_{N / 2-1} \frac{J_{\ell_{k}+1}\left(j_{N / 2-1}\right)}{J_{\ell_{k}}\left(j_{N / 2-1}\right)}}\right]
$$

and the remainder of the proof of Theorem 1.2 consists in showing that the infimum on righthand side of (21) is achieved for $k=2$ independently on $N$. In fact the constant $C_{N}$ defined in Theorem 1.2 coincides with

$$
\left.\left[\left(\frac{j_{N / 2-1}^{2}}{2 N \omega_{N} G_{N}^{2}}\right) \frac{\ell_{k}^{2}-\frac{N^{2}}{4}}{\ell_{k}+\frac{N}{2}-j_{N / 2-1} \frac{J_{\ell_{k}+1}\left(j_{N / 2-1}\right)}{J_{\ell_{k}}\left(j_{N / 2-1}\right)}}\right]\right|_{k=2} .
$$

In principle, minimizing the righthand side of (21) is elementary. However it is worth providing the details, since the proof involves the usage of several nontrivial properties of the Bessel functions.

The next Lemma concludes the proof of Theorem 1.2. In what follows we use the notion of convex sequence:

Definition 2.2. We say that a sequence $\left\{\alpha_{k}\right\}_{k \in \mathbb{N}}$ of real numbers is convex (concave) for $k \geq k_{0}$ if $\alpha_{k+1}-2 \alpha_{k}+\alpha_{k-1} \geq 0(\leq 0)$ when $k \geq k_{0}+1$.

Lemma 2.3. For all $N \geq 2$, let $k \geq 2, \ell_{k}=k+N / 2-1$, and

$$
\mathcal{Q}_{k}=\left(\frac{j_{N / 2-1}^{2}}{2 N \omega_{N} G_{N}^{2}}\right) \frac{\ell_{k}^{2}-\frac{N^{2}}{4}}{\ell_{k}+\frac{N}{2}-j_{N / 2-1} \frac{J_{\ell_{k}+1}\left(j_{N / 2-1}\right)}{J_{\ell_{k}}\left(j_{N / 2-1}\right)}} .
$$

We have $\mathcal{Q}_{k} \leq \mathcal{Q}_{k+1}$.

Proof of Lemma 2.3. First we prove that, for any given value $N \geq 2$, the sequence $\left\{\frac{J_{\ell_{k}+1}\left(j_{N / 2-1}\right)}{J_{\ell_{k}}\left(j_{N / 2-1}\right)}\right\}_{k \in \mathbb{N}}$ is positive, decreasing, vanishing, and convex for $k \geq 2$. Denoting by $z_{N}=j_{N / 2-1}$, the claim follows at once from the continued fraction representation (see $[1, \S 9.1 .73]$ )

$$
\frac{J_{\ell_{k}+1}\left(z_{N}\right)}{J_{\ell_{k}}\left(z_{N}\right)}=\frac{1}{\frac{2\left(\ell_{k}+1\right)}{z_{N}}-\frac{1}{\frac{2\left(\ell_{k}+2\right)}{z_{N}}-\frac{1}{\frac{2\left(\ell_{k}+3\right)}{z_{N}}-\ddots}}}
$$


In fact, after observing that $\frac{2\left(\ell_{k}+1\right)}{z_{N}}>1$ (see [4, 20]) it is easy to deduce that the sequence $\left\{\frac{J_{\ell_{k}+1}\left(j_{N / 2-1}\right)}{J_{\ell_{k}}\left(j_{N / 2-1}\right)}\right\}_{k \in \mathbb{N}}$ is positive decreasing and vanishing. It remains to prove the convexity and we begin observing that if a sequence $\left\{\alpha_{k}\right\}_{k \in \mathbb{N}}$ is concave then $\left\{\alpha_{k}^{-1}\right\}_{k \in \mathbb{N}}$ is convex, hence

$$
\frac{1}{\frac{2\left(\ell_{k}+1\right)}{z_{N}}}, \quad k \in \mathbb{N}
$$

is convex for $k \geq 2$ but also

$$
\frac{1}{\frac{2\left(\ell_{k}+1\right)}{z_{N}}-\frac{1}{\frac{2\left(\ell_{k}+2\right)}{z_{N}}}}, \quad \text { and } \quad \frac{1}{\frac{2\left(\ell_{k}+1\right)}{z_{N}}-\frac{1}{\frac{2\left(\ell_{k}+2\right)}{z_{N}}-\frac{1}{\frac{2\left(\ell_{k}+3\right)}{z_{N}}}}} \quad k \in \mathbb{N}
$$

are convex for $k \geq 2$, as well as any truncation of the continued fraction (22). By approximation we deduce that $\left\{\frac{J_{\ell_{k}+1}\left(j_{N / 2-1}\right)}{J_{\ell_{k}}\left(j_{N / 2-1}\right)}\right\}_{k \in \mathbb{N}}$ is a convex sequence for $k \geq 2$. Eventually we deduce that, for any $N$ fixed, $\mathcal{Q}_{k}$ is a convex sequence for $k \geq 2$ since it is the ratio between a positive convex sequence $\left\{\left(\frac{z_{N}^{2}}{2 N \omega_{N} G_{N}^{2}}\right)\left(\ell_{k}^{2}-\frac{N^{2}}{4}\right)\right\}_{k \in \mathbb{N}}$ and a positive concave sequence $\left\{\ell_{k}+\frac{N}{2}-z_{N} \frac{J_{\ell_{k}+1}\left(z_{N}\right)}{J_{\ell_{k}}\left(z_{N}\right)}\right\}_{k \in \mathbb{N}}$.

The convexity of $\mathcal{Q}_{k}$ for $k \geq 2$ implies the increasing monotonicity of $\mathcal{Q}_{k}$ for $k \geq 2$ if and only if $\mathcal{Q}_{2} \leq \mathcal{Q}_{3}$. In view of the recurrence relations of the Bessel functions $[1, \S 9.1 .27]$, we have

$$
\begin{gathered}
\frac{J_{\ell_{2}}\left(z_{N}\right)}{J_{\ell_{1}}\left(z_{N}\right)}=\frac{N}{z_{N}}, \\
\frac{J_{\ell_{3}}\left(z_{N}\right)}{J_{\ell_{2}}\left(z_{N}\right)}=\frac{N+2}{z_{N}}-\frac{z_{N}}{N},
\end{gathered}
$$

and

$$
\frac{J_{\ell_{4}}\left(z_{N}\right)}{J_{\ell_{3}}\left(z_{N}\right)}=\frac{N+4}{z_{N}}-\left(\frac{N+2}{z_{N}}-\frac{z_{N}}{N}\right)^{-1} .
$$

After a tedious but straightforward computation we get $\mathcal{Q}_{2}<\mathcal{Q}_{3}$ if and only if

$$
\left(z_{N}^{2}-2\right) N^{2}+5 z_{N}^{2} N-2 z_{N}^{4}>0 .
$$

It is not difficult to prove that the last inequality holds true for large values of $N$, in view of the following upper and lower bounds on the first zero of Bessel functions $($ see $[4,20])$

$$
\frac{N}{2}-1 \leq z_{N} \leq \sqrt{\frac{N}{2}}\left(\sqrt{\frac{N}{2}+1}+1\right)
$$


In fact plugging (24) in (23) we have

$$
\begin{aligned}
& \left(z_{N}^{2}-2\right) N^{2}+5 z_{N}^{2} N-2 z_{N}^{4} \\
& =z_{N}^{2}\left(N^{2}-2 z_{N}^{2}+2 N\right)+N\left(3 z_{N}^{2}-2 N\right) \\
& \geq z_{N}^{2}\left(N^{2}-N(\sqrt{N / 2+1}+1)^{2}+2 N\right)+N\left(3(N / 2-1)^{2}-2 N\right) \\
& =\frac{N z_{N}^{2}}{2}\left(N-2^{3 / 2} \sqrt{N+2}\right)+\frac{3}{4} N(N-6)\left(N-\frac{2}{3}\right)
\end{aligned}
$$

and it is not difficult to prove that the last quantity is increasing for $N \geq 10$ and positive for $N=10$ therefore positive for $N \geq 10$.

For the remaining values $2 \leq N \leq 9$, it is elementary to check inequality (23) using the following table

\begin{tabular}{|c|c|}
\hline$N$ & $z_{N} \equiv j_{N / 2-1}$ \\
\hline 2 & $\approx 2.404826$ \\
3 & $\pi$ \\
4 & $\approx 3.831706$ \\
5 & $\approx 4.4934095$ \\
6 & $\approx 5.135622$ \\
7 & $\approx 5.763459$ \\
8 & $\approx 6.380162$ \\
9 & $\approx 6.987932$ \\
\hline
\end{tabular}

and the proof is complete.

According to the compatibility condition (19), by construction it is clear that

$$
\liminf _{t \rightarrow 0} \frac{\delta P(\Omega(t))}{\delta \lambda(\Omega(t))}=C_{N}
$$

provided

$$
V(\xi)=Y_{2}(\xi) \quad \text { and } \quad \int_{\mathcal{S}^{N-1}} A(\xi) d \sigma_{\xi}=(1-N)
$$

\section{REFERENCES}

[1] M. Abramowitz, I. Stegun, A. Handbook of mathematical functions with formulas, graphs, and mathematical tables. National Bureau of Standards Applied Mathematics Series, 1964. MR0167642

[2] A. Alvino, V. Ferone, C. Nitsch, A sharp isoperimetric inequality in the plane, J. Eur. Math. Soc. (JEMS) 13 (2011), no. 1, 185-206. MR2735080

[3] B. Brandolini, C. Nitsch, C. Trombetti, An upper bound for nonlinear eigenvalues on convex domains by means of the isoperimetric deficit, Arch. Math. (Basel) 94 (2010), no. 4, 391-400. MR2643973

[4] Ll. G. Chambers, An upper bound for the first zero of Bessel functions, Math. Comp. 38 (1982), no. 158, 589-591. MR0645673

[5] M. Cicalese, G. P. Leonardi, A selection principle for the sharp quantitative isoperimetric inequality, preprint.

[6] M. Cicalese, G. P. Leonardi, Best constants for the isoperimetric inequality in quantitative form, to appear on J. Eur. Math. Soc.

[7] L. Esposito, N. Fusco, C. Trombetti, A quantitative version of the isoperimetric inequality: the anisotropic case, Ann. Sc. Norm. Super. Pisa Cl. Sci. (5) 4 (2005), no. 4, 619-651. MR2207737

[8] A. Figalli, F. Maggi, A. Pratelli, A mass transportation approach to quantitative isoperimetric inequalities, Invent. Math. 182 (2010), no. 1, 167-211. MR2672283 
CARLO NITSCH

[9] B. Fuglede, Stability in the isoperimetric problem for convex or nearly spherical domains in $\mathbf{R}^{n}$, Trans. Amer. Math. Soc., 314 (1989), 619-638. MR942426

[10] N. Fusco, F. Maggi, A. Pratelli, The sharp quantitative isoperimetric inequality, Ann. of Math. (2) 168 (2008), no. 3, 941-980. MR2456887

[11] N. Fusco, F. Maggi, A. Pratelli, Stability estimates for certain Faber-Krahn, isocapacitary and Cheeger inequalities, Ann. Sc. Norm. Super. Pisa Cl. Sci. (5) 8 (2009), no. 1, 5171. MR2512200

[12] D. Gilbarg, N. S. Trudinger, Elliptic partial differential equations of second order, Classics in Mathematics. Springer-Verlag, Berlin, 2001. MR1814364

[13] A. Henrot, Extremum problems for eigenvalues of elliptic operators, Frontiers in Mathematics. Birkhuser Verlag, Basel, 2006. MR2251558

[14] A. Henrot, M. Pierre, Variation et optimisation de formes. Une analyse géométrique. Mathématiques \& Applications, vol. 48, Springer, 2005. MR2512810

[15] S. Kesavan, Symmetrization 83 applications. Series in Analysis, 3. World Scientific Publishing Co. Pte. Ltd., Hackensack, NJ, 2006. MR2238193

[16] A. D. Melas, The stability of some eigenvalue estimates, J. Differential Geom. 36 (1992), no. 1, 1933. MR1168980

[17] C. Müller, Spherical harmonics. Lecture Notes in Mathematics, 17 Springer-Verlag, BerlinNew York 1966. MR0199449

[18] A. Novruzi, M. Pierre, Structure of shape derivatives, J. Evol. Equ. 2 (2002), no. 3, 365-382. MR1930612

[19] L. E. Payne, H. F. Weinberger, Some isoperimetric inequalities for membrane frequencies and torsional rigidity, J. Math. Anal. Appl. 2 (1961), 210-216. MR0149735

[20] C. K. Qu, R. Wong, "Best possible" upper and lower bounds for the zeros of the Bessel function $J_{\nu}(x)$, Trans. Amer. Math. Soc. 351 (1999), no. 7, 2833-2859. MR1466955

[21] J. Sokolowski and J. P. Zolésio, Introduction to shape optimization. Shape sensitivity analysis. Springer Series in Computational Mathematics, vol. 16. Springer-Verlag, Berlin, 1992. MR1215733

E-mail address: c.nitsch@unina.it 